Uso del Brain Gym y su incidencia en el desarrollo cognitivo de niños y niñas.

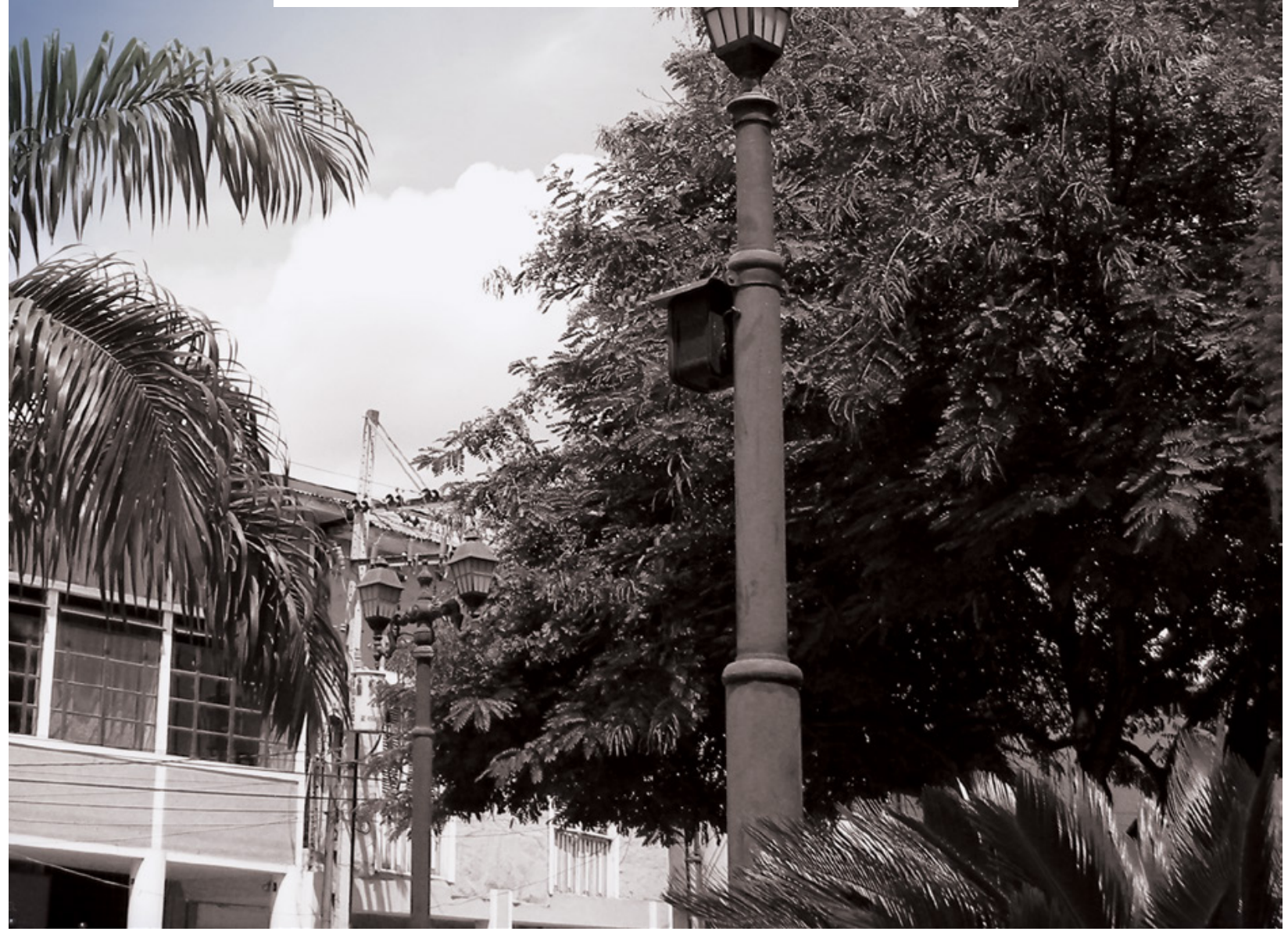




\title{
USO DEL BRAIN GYM Y SU INCIDENCIA EN EL DESARROLLO COGNITIVO DE NIÑNOS Y NIÑNA.
}

\section{USE OF BRAIN GYMNASTICS AND ITS IMPACT ON THE COGNITIVE DEVELOPMENT OF CHILDREN}

\begin{abstract}
RESUMEN
La presente investigación se realizó como respuesta a la necesidad de los docentes de actividades y métodos dinámicos e innovadores, que fortalecen la interrelación con los niños y niñas. La metodología empleada fue de enfoque mixto, una investigación cuasi-experimental con alcance exploratorio y explicativo, con predominio cuali-cuantitativo; ejecutada en el Centro de Educación Inicial "El Paraíso de los Niños" durante el periodo 2016-2017 con grupos del Subnivel 2; donde se pretendió determinar la incidencia del Brain Gym en el desarrollo cognitivo. Al establecer las tabulaciones se evidencia que las conclusiones fueron favorables y la hipótesis planteada fue demostrada. De esta manera la herramienta metodológica propuesta recopiló un sin número de ejercicios de Gimnasia Cerebral los mismos que estimularon algunas regiones del cerebro, para así lograr un desarrollo integral, pero en especial las relacionadas con las funciones mentales. Los resultados obtenidos en el pre test y pos test permitieron llegar a la conclusión que los ejercicios propuestos incrementaron el nivel cognitivo de los niños.
\end{abstract}

PALABRAS CLAVE: Cerebro; funciones cerebrales; gimnasia; hemisferios.

Copyright @ Revista San Gregorio 2019. ISSN 1390-7247; eISSN: 2528-7907 @

\begin{abstract}
The present investigation was carried out in response to the teachers' need for dynamic and innovative activities and methods, which strengthen the interrelation with children. The methodology used was a mixed approach, a quasi-experimental research with exploratory and explanatory scope, with a qualitative-quantitative predominance; executed in the Initial Education Center "El Paraíso de los Niños" during the period 2016-2017 with groups of Sub-level 2; where it was intended to determine the incidence of Brain Gym in cognitive development. When establishing the tabulations, it is evident that the conclusions were favorable and the hypothesis was demonstrated. In this way, the proposed methodological tool collected a number of exercises in Brain Gymnastics that stimulated certain regions of the brain, in order to achieve an integral development, but especially those related to mental functions. The results obtained in the pre-test and post-test allowed to reach the conclusion that the proposed exercises increased the cognitive level of the children.
\end{abstract}

KEYWORDS: Brain; brain functions; gymnastics; hemispheres.

Copyright @ Revista San Gregorio 2019. ISSN 1390-7247; eISSN: 2528-7907

Cinthia Tatiana Moreira Cedeño

A Centro de Educación Inicial Fiscal “Augusta Ugalde Alcívar".

- tatysmore@hotmail.com

ARTÍCULO RECIBIDO: 7 DE JUNIO DE 2018

ARTÍCULO ACEPTADO PARA PUBLICACIÓN: 7 DE JUNIO DE 2019

ARTÍCULO PUBLICADO: 30 DE JUNIO DE 2019 


\section{INTRODUCCIÓN}

La presente investigación se refiere al uso del Brain Gym y su influencia en el desarrollo cognitivo de los niños y niñas de Inicial 2 del Centro de Educación Inicial Fiscal "El Paraíso de los Niños" de la ciudad de Portoviejo, se planteó este tema ya que dicha institución muestra que existen actividades lúdicas que despliegan un sin número de habilidades; sin embargo, se está dejando a un lado el desarrollo de funciones cognitivas para así poder alcanzar procesos mentales superiores indispensables para su vida futura.

El Brain Gym o como algunas personas la conocen "ejercicios mentales" son un conjunto de actividades previstas, las mismas que generan procesos mentales por medio de la activación de puntos energéticos, acelerando el desarrollo y las capacidades de los que lo utilizan. Los avances científicos demuestran que el cerebro y el cuerpo están íntimamente ligados, por lo que al realizar estos ejercicios se activarán y potenciará el funcionamiento de nuestro cerebro y por ende de habilidades y destrezas mentales

En las aulas a pesar de que existen una gran cantidad de docentes preparados en el ámbito que imparten, son muy pocas las veces que se preparan ejercicios para estimular el cerebro de los niños y niñas. Pero hay que considerar que la gimnasia cerebral no solo puede ser utilizada para esta edad, sino para todo tipo de personas ya que el objetivo principal es el de mejorar las capacidades intelectuales, físicas y emocionales, además, la atención, disciplina y comportamiento.

Es por lo antes planteado que se considera pertinente aplicar programas en las aulas de clases que permitan esta activación cerebral, ya que, de esta manera, se obtiene resultados beneficiosos para nuestros niños y niñas. Así ellos tendrán un mejor control emocional, existiría un desarrollo físico notable acorde a su edad y, por ende, el perfeccionamiento de las habilidades y destrezas primordiales para su desarrollo y aprendizaje.

\section{ANTECEDENTES INVESTIGATIVOS}

La presente investigación está sustentada en el análisis y revisión de investigaciones previas donde las variables planteadas por los autores están en íntima relación con las del trabajo actual, es así que se menciona en la Tabla 1 (Ver en Anexos).

Al analizar cada uno de los estudios planteados no solo en el Ecuador, sino también en Argentina, se puede concluir que a pesar de ser un aspecto nuevo que está ingresando paso a paso al nivel educativo, existen investigaciones que ponen a prueba la teoría plantea$\mathrm{da}$, demostrando que las actividades relacionadas con el Brain Gym son un aporte valioso para las diferentes áreas de desarrollo de los niños, niñas y adolescentes.

Si comparamos la presente investigación con aquellas realizadas, se puede mencionar que no existe ninguna cuyas variables sean las planteadas, es decir, no existen investigación sobre el Brain Gym y el desarrollo cognitivo. Por lo que no podría realizarse una confrontación de los resultados. Sin embargo, al igual que otras investigaciones relacionadas con el Brain Gym, en esta se obtuvo resultados favorables, donde los niños y niñas mejoraron al realizar las actividades relacionadas con la atención, percepción y lenguaje cuyo valor según la escala propuesta fue de muy bueno.

\section{JUSTIFICACIÓN DE LA INVESTIGACIÓN}

Haciendo un recorrido por la evolución de la educación, iniciando con el conductismo donde el docente ponía en práctica el uso de metodologías conductistas y el estudiante era considerado receptor pasivo, siguiendo con el constructivismo el cual ubica los aportes de autores como Piaget, Vygotsky, Ausubel, Bronfenbrenner y con sus investigaciones sobre la zona de desarrollo próximo, conceptos de asimilación y acomodación, aprendizaje significativo y teorías de sistemas ambientes que permiten entender que los procesos de aprendizaje se generan a partir de conocimientos previos, y con la influencia del medio en que se desenvuelven; hasta la actualidad con una gran cantidad de pedagogos que día a día implementan nuevos métodos que ayudan 
a mejorar el aprendizaje, se puede notar que la educación cada vez pretender el desarrollo máximo de los seres humanos. (Schunk D. H., 2012).

Pues, las bases con las que el Currículo de Educación inicial trabaja son aquellas resultantes de los trabajos de investigación de: (Vygotski, 1979) década de los 30, (Bronfenbrenner U. , 1987), (Rogoff, 1993) y (Tinajero A. y Mustard, 2011) donde sustentan que los niños y niñas necesitan ambientes estimulantes y positivos que favorezcan el desarrollo y aprendizaje de los infantes.

Según el documento Bienes públicos regionales para la atención de la primera infancia (CEPALGIZ) las neurociencias muestran que, si bien el sistema nervioso puede remodelar los contactos entre neuronas y la eficiencia de sus conexiones, permitiendo siempre generar alguna modificación (neuroplasticidad), existen períodos críticos o sensibles, en que las redes neuronales tienen una excepcional susceptibilidad a los efectos del medio ambiente y la experiencia (interacciones sociales y con objetos). Las etapas tempranas de la vida constituyen un período crítico o sensible. En este período, las experiencias que se establecen con el entorno influirán no sólo en la forma de construir la identidad, sino también en cómo se estructure y funcione el cerebro, sentándose las bases del aprendizaje y la socialización (Ecuador, 2014).

A pesar de existir aportes como los antes mencionados y contar con un Currículo que plantea el desarrollo de ámbitos como el del desarrollo cognitivo, los docentes de Educación Inicial en ocasiones no proporcionan los estímulos adecuados y necesarios para que los niños y niñas desarrollen sus capacidades y por ende las actividades relacionadas a la cognición, y es así que los maestros consideran adecuado la presencia de problemas de lenguaje, atención, memoria, percepción y concentración en las aulas de clases. Es aquí donde se deben implementar actividades y herramientas innovadoras dentro y fuera del salón de clases como la gimnasia cerebral permitiendo la ejecución de actividades que estimulen las conexiones neuronales y por ende la ejecución de las funciones mentales superiores mencionadas anteriormente ya que es en el campo de acción, en el día a día, en el juego y trabajo con los niños y niñas que se observan las complicaciones y aciertos de estos pequeños y donde si bien es cierto los docentes no tienen la potestad de diagnosticar estos problemas, si pueden ser los primeros veedores para derivar al profesional correspondiente y solucionar las falencias encontradas.

Todo esto exige una gran importancia en el desarrollo integral de los niños y niñas, por lo que la presente investigación denota gran importancia en su realización para poder conocer sobre esta innovadora técnica de estimulación cerebral llamada Brain Gym, misma que ha pasado desapercibida. Por otra parte, tiene como fin elevar los procesos mentales de los niños y niñas con actividades a realizarse dentro y fuera del aula. Su puesta en escena servirá como material de apoyo para los docentes del C.E.I Fiscal "El Paraíso de los niños" para que tengan una técnica innovadora y vinculen la estimulación del cerebro con el desarrollo y aprendizaje de los niños y niñas, permitiendo que los dos hemisferios cerebrales se unan para trabajar como uno solo.

Esta investigación es útil ya que permite a los docentes que además de las actividades que realizan diariamente los niños y niñas, se mantengan ocupados, relajados y concentrados, pero sobre todo preparados para captar de manera rápida las nuevas experiencias y por ende el aprendizaje. Como dato extra, el maestro puede apoderarse de esta técnica incluyéndola en la planificación diaria ya que beneficia adicionalmente a la resolución de problemas cotidianos y a la salud emocional de los párvulos.

Son estas las razones por las que se justifica la investigación ya que, a través de la gimnasia cerebral desde edades tempranas, los docentes tienen técnicas innovadoras en su arsenal educativo y los niños y niñas desarrollan procesos mentales en beneficio de su desarrollo y aprendizaje.

\section{OBJETIVOS}

\section{Objetivo General.}

Determinar la incidencia del uso del Brain Gym en el desarrollo cognitivo de los niños y niñas del Subnivel Inicial 2 del Centro de Educación Inicial "El Paraíso de los Niños" de la ciudad de Portoviejo. 


\section{ОвJETIVOS ESPECÍfICOS.}

- Caracterizar el nivel de desarrollo cognitivo de los niños y niñas del Subnivel Inicial 2 del Centro de Educación Inicial Fiscal "El Paraíso de los Niños".

-Diseñar una herramienta pedagógica que considere la aplicación de la Gimnasia cerebral como parte de las actividades iniciales del proceso didáctico.

\section{METODOLOGÍA}

La presente investigación tiene las siguientes características: Enfoque mixto cualitativo-cuantitativo. La recolección, análisis e interpretación de los resultados cuantitativos fueron obtenidos de la aplicación de un pre test y pos test, en donde los datos numéricos y la observación permitieron una relación entre las variables y el objeto de estudio, mismo que es la gimnasia cerebral en el desarrollo cognitivo.

Para la caracterización de cada uno de los aspectos del desarrollo cognitivo, es decir, memoria, concentración, percepción, lenguaje y atención; se elaboró una matriz valorativa tipo Liker que contenía como indicadores las destrezas del Currículo de Educación Inicial relacionadas con los aspectos cognitivos antes mencionados.

Se planteó como hipótesis alterna que el uso del Brain Gym incide en el desarrollo cognitivo de los niños y niñas de Inicial 2 del Centro de Educación Inicial Fiscal "El Paraíso de los Niños" de la ciudad de Portoviejo. Y como hipótesis nula que, el uso del Brain Gym no incide en el desarrollo cognitivo de los niños y niñas del Inicial 2 del Centro de Educación Inicial Fiscal "El Paraíso de los Niños" de la ciudad de Portoviejo.

Es así que se analiza la población de niños de cuatro años misma que es representada por 188 niños y niñas de los cuales se tomó una muestra de 60 párvulos del Centro de Educación Fiscal "El Paraíso de los niños", dentro del ámbito educativo se conectan directamente con las necesidades de los destinatarios y destinatarias.

El nivel es explicativo porque alcanza una relación de dependencia que existe entre las variables, para lograr este nivel se realizaron procesos exploratorios y descriptivos.
Tiene un alcance cuasi experimental, donde se observaron la gran mayoría de los pasos o procedimientos de una experimental, pero con la diferencia de que en este caso no se escogió de manera aleatoria del grupo que se estudia. Es decir, se estuvo comprobando la relación entre las variables y además se presentó un programa aplicado donde se realizaron comparaciones sobre su eficacia o no.

Este programa es la propuesta "Brain Gym como herramienta pedagógica para el apoyo del desarrollo cognitivo" misma que consiste en aplicar varios ejercicios de Brain Gym durante las actividades iniciales, para así mejorar las actividades planteadas por la docente investigadora.

\section{RESULTADOS}

Una vez que se conoció la población se procedió a escoger la muestra de manera aleatoria escogiendo dos paralelos de los seis existentes en el Centro de Educación Inicial los mismos que corresponden al paralelo "D" y el paralelo " $E$ ” ambos con 30 niños y niñas, un paralelo fue el grupo de control y el otro el grupo experimental.

Luego de haber aplicado el instrumento de evaluación a los niños y niñas los resultados se tabularon y organizaron por medio de tablas para ser procesados en términos de medidas descriptivas como lo mencionaremos a continuación: distribución de frecuencias, porcentajes, medias aritméticas, desviación y puntaje $\mathrm{Z}$.

Al comparar el valor de $\mathrm{Z}$ calculado y el valor de $\mathrm{Z}$ teórico $\mathrm{z} \_\mathrm{c}<\mathrm{z}$ _t $; 9.4<1,96$. Podemos observar que zc $=9.4$ está en la zona de aceptación de la hipótesis de investigación, lo cual nos lleva a aceptar la hipótesis alternativa (Hi) de investigación y rechazar la hipótesis nula (Ho).

En otras palabras, el desarrollo cognitivo de los niños y niñas que utilizaron el Brain Gym como herramienta didáctica, superaron en gran proporción al grupo de niños y niñas que no utilizo el manual de ejercicios prácticos.

En las actividades relacionadas con la percepción del Post test, se obtuvieron los siguientes porcentajes: en el grupo experimental la escala de muy bueno muestra un $41 \%$ mientras que el grupo de control presenta un $42 \%$ en la escala insuficiente, lo que comprue- 
ba que hubo un avance en el grupo experimental.

En el nivel de desarrollo de los niños y niñas en las actividades de atención se obtienen los siguientes resultados del post test: $57 \%$ en la escala muy bueno como valor más elevado del grupo experimental y $52 \%$ en la escala insuficiente del grupo control. Esto demuestra que los porcentajes mejoraron en el grupo experimental.

Para el nivel de desarrollo de los niños y niñas en las actividades relacionadas con la concentración, se observó cómo porcentajes más elevados los siguientes: grupo experimental $37 \%$ en la escala bueno y en el grupo de control $49 \%$ en la escala insuficiente. Lo que indica que las actividades relacionadas con la concentración mejoraron en el grupo experimental.

De las actividades relacionadas con la memoria aplicada a los niños y niñas, se obtuvo: $41 \%$ en la escala bueno para el grupo experimental y el $48 \%$ en la escala de insuficiente para el grupo de control. Esto indica que el nivel de desarrollo de las actividades de la memoria mejoró en el grupo experimental.

Con respecto a las actividades relacionadas con el lenguaje, se obtuvieron los siguientes porcentajes: grupo experimental $41 \%$ en la escala muy bueno; mientras que en el grupo de control se observa $42 \%$ en la escala insuficiente. Lo que indica que existe un aumento considerable en el grupo experimental.

\section{DISCUSIÓN}

(Hatfield, 2006) "considera al Brain Gym como un conjunto de actividades divertidas, veloces y energéticas que optimizan el aprendizaje, según sus proponentes, estas actividades preparan a cualquier alumno para reforzar destrezas de coordinación y pensamiento". (p.260).

Es desde ahí que se le da gran importancia al movimiento para el desarrollo y aprendizaje; su constante aplicación permite la estimulación de conexiones neuronales y, por tanto, mayor riqueza en su estructura. Cuando esto sucede se activan las neuronas produciendo una sustancia que incrementa la velocidad en la transmisión de los impulsos nerviosos (mielina) haciendo más rápida la transmisión de la información.
En la presente investigación que hace referencia al Brain Gym y el desarrollo cognitivo, se planteó como pregunta inicial ¿Cómo incide el uso del Brain Gym en el desarrollo cognitivo de los niños y niñas de Inicial 2 del Centro de Educación Inicial fiscal "el Paraíso de los Niños" de la ciudad de Portoviejo? Para poder despejar esta interrogante se analizaron datos bibliográficos, se aplicaron técnicas de recolección de datos, pero sobre todo se diseñó una herramienta pedagógica misma que planteaba actividades iniciales dentro del proceso didáctico, es decir, se establecieron ejercicios de Brain Gym.

Antes de aplicar dichos ejercicios, se evaluó tanto al grupo experimental como al de control para tener datos referentes al pre test, para luego proceder a ejecutar las actividades solo con el grupo experimental, el grupo de control realizó las mismas actividades relacionadas con las destrezas del Currículo de Educación Inicial, la diferencia está en que no se aplicó la propuesta cuyo nombre es "Brain Gym como herramienta pedagógica para el apoyo del desarrollo cognitivo de los niños y niñas"

Es así que se da la aplicación de la propuesta con los siguientes lineamientos: iniciar con la ingesta de medio vaso de agua o un cuadrito de chocolate (de forma salteada), esto con el fin de estimular los puntos energéticos del cuerpo. Posteriormente se procedió a realizar un ejercicio de Brain Gym, explicárselo a los niños y realizarlo junto a ellos. Estos ejercicios fueron intercalándose para evitar caer en la monotonía.

Al hablar de Brain Gym y movimientos es importante reconocer la utilización de los brazos y piernas y para poder explicar estos movimientos existen tres dimensiones muy importantes que (Dennison, 1997) las ha descrito de esta manera, como ejercicios de la gimnasia cerebral. (Dennison, 1997) e (Ibarra, 2007) proponen en sus investigaciones un sin número de ejercicios para trabajar la gimnasia cerebral, a continuación se detallarán los más relevantes. Estos ejercicios fueron: marcha cruzada, 8 perezoso, doble garabato, el elefante, giros del cuello, marcha cruzada en el suelo, la cobra, el búho, activación del brazo, bombeo de pantorrilla, balanceo de gravedad, botones cerebrales, botones de tierra, botones de equilibrio, botones de espacio, bostezo de energía, sombrero de pensar, 
espantado, respiración abdominal, el perrito, el pinocho

Después de estas actividades, se continuó con la planificación habitual de acuerdo a los lineamientos del Ministerio de Educación. Cabe desatacar que esta propuesta pedagógica se aplicó solo al grupo experimental durante 9 semanas.

Terminadas las 9 semanas se volvió a evaluar a los niños y niñas tanto del grupo experimental como de control dando como resultado que el grupo experimental mejoró la realización de actividades relacionadas con el desarrollo cognitivo, es decir, actividades de percepción, concentración, atención, lenguaje y memoria. Esto se evidencia en los resultados derivados de la matriz valorativa mismos que indicaban que en el pre test los dos grupos estudiados muestran porcentajes altos en la escala de insuficiente y regular, sin embargo, al aplicar la propuesta pedagógica se notó que las actividades relacionadas con percepción, concentración y lenguaje elevaron su valor significativamente. Mientras que en el lenguaje y memoria se mantuvieron los valores e incluso disminuyeron.

Esto nos da a notar que al aplicar la propuesta pedagógica se mejoró las actividades relacionadas con el desarrollo cognitivo de los niños y niñas. Pero si bien es cierto se mejoraron algunos del proceso del desarrollo cognitivo hay otros que no, esto evidenció que hubo falencias que no permitieron que la investigación sea del todo precisa.

Es por esta razón que como aspectos a mejorar o en caso de aplicar esta propuesta en posteriores momentos se recomienda que la implementación se dé desde el inicio de clases, incluyendo el periodo de adaptación, que se realicen durante las primeras horas de la jornada de clases y que las maestras conozcan detenidamente como se realiza cada ejercicio para que así cumpla el propósito establecido.

Con todo lo expuesto se puede concluir que la aplicación de los ejercicios de Brain Gym inciden en el desarrollo cognitivo de los niños y niñas, por lo que es una temática importante e innovadora para aplicar diariamente en el proceso de formación de los párvulos. Así mismo que dentro de los beneficios del Brain Gym tenemos:

-Mantiene la memoria lúcida.

-Pensamiento activo.

-Incrementa la creatividad

-Manejar el estrés y desarrollo personal

-Integra mente/cuerpo.

-Ayuda a los niños y niñas con lento aprendizaje, hiperactividad, síndrome de Down, entre otros.

-Ayuda a la concentración y focalización.

-Coordinación física y equilibrio

-Habilidades de comunicación y desarrollo lingüístico

-Fortalece habilidades y procesos de aprendizaje

-Ayuda a mejorar en matemáticas

-Leer y escribir con facilidad

-Mejora el comportamiento y la actitud positiva.

-Se crean nuevos caminos neuronales.

Por último, hay que recordar las funciones mentales que se trabajaron en esta investigación y la relevancia que tiene en el desarrollo integral de los niños y niñas.

\section{PeRCEPCIÓN}

"La percepción resulta de la actuación de una serie de procesos mediatizados neurofisiológicamente que desarrollan y transforman activamente, a lo largo del tiempo, las representaciones sistemáticas de los estímulos ambientales" (Pérez-Cortés, 2003).

\section{Atención}

Se la puede considerar como el proceso que permite controlar las actividades del individuo para planificar y adaptarse al ambiente, desde el punto de vista del estructuralismo se la ve como un estado de consciencia y desde el funcionalismo es una función activa del individuo cuyo fin es la adaptación.

\section{CONCENTRACIÓN}

Se puede considerar a la concentración como la habilidad de retener atención durante un periodo más largo utilizando la mayor cantidad de sentidos y sin distraerse de los estímulos externos. La concentración es una destreza aprendida, de reaccionar pasivamente o de no distraerse ante estímulos irrelevantes. "La concentración también significa 
el estar totalmente aquí y en el ahora, en el presente" (Schmid, 1991).

\section{MEMORIA}

Se la puede considerar como la función que tiene el cerebro para evocar aquello que ha pasado recordando las experiencias anteriores y creando nuevas de una manera clara. Durante este proceso se necesitan una codificación de las experiencias y esto va ser mucho más efectiva dependiendo de dos factores importantes como son la atención y la motivación.

Esta información se encuentra en algunas zonas corticales y depende de la capacidad de cada persona ya que unas tendrán mejor manera de seleccionar la información útil y desechar lo innecesario, es decir, se almacena lo importante y lo otro se deja a un lado mientras que en personas con poca memoria se almacena una gran cantidad por lo que no hay una buena selección de la información apropiada.

(Rey, 2006) Menciona 3 fases y 3 formas de exploración:

- Aprendizaje: Recepción y registro sensorial de la información.

- Almacenamiento: Computa su codificación cerebral.

- Recuerdo: Evocación y reconocimiento.

\section{LENGUAJE}

Son todos aquellos sonidos o gráficos que permiten entablar un diálogo fluido entre las personas, que se origina durante la evolución de las especies, esta evolución es tanto morfológica como funcional.

"El centro del lenguaje se encuentra en el hemisferio izquierdo, dentro de la corteza cerebral las áreas encargadas de su ejecución son Área de Wernicke, área de Broca, Fascículo Longitudinal Superior y giro angular" (Hall, 2011).

\section{CONCLUSIONES}

Una vez realizada la investigación se puede concluir que:

La incidencia del Brain Gym en el desarrollo cognitivo se pudo evidenciar por medio de los resultados arrojados en la investigación, ya que se observó que en el grupo experimental del post test hubo un incremento considerable en las actividades relacionadas con la percepción, concentración y atención.

Se pudo caracterizar el nivel de desarrollo de las actividades relacionadas al aspecto cognitivo que presentan los párvulos del subnivel 2 del Centro de Educación Inicial "El Paraíso de los Niños”. De la siguiente manera: en el pre test los dos grupos presentaron valores elevados en la escala de insuficiente y regular. En el post test, el grupo control alcanza como valores más notorios los ubicados en la escala de insuficiente con los porcentajes: percepción $42 \%$, atención $52 \%$, concentración 49\%, memoria $48 \%$, lenguaje $42 \%$; mientras que en el grupo experimental el nivel de las actividades relacionadas con el desarrollo cognitivo se ubican en la escala de muy bueno, teniendo así percepción 41\%, atención 57\%, lenguaje $41 \%$, a excepción de las actividades relacionadas con la memoria que tiene una escala de bueno con un $41 \%$ y la concentración que es regular con $37 \%$ en el grupo experimental y $49 \%$ grupo de control.

Para establecer la alternativa de solución del problema con el diseño de la "Brain Gym como herramienta pedagógica para el apoyo del desarrollo cognitivo" se contemplaron las disposiciones del Referente Curricular Nacional, así como los lineamientos metodológicos para el nivel inicial sin descuidar las destrezas planteadas para este nivel y en conjunto con actividades que potencien el desarrollo cognitivo como la memoria, concentración, percepción, atención y lenguaje. 


\section{REFERENCIAS BIBLIOGRÁFICAS}

Balcazar, A. L. (2012). Importancia de la Gimnasia Cerebral en el desarrollo de la creatividad de los niños. Ambato, Ecuador.

Bronfenbrenner, U. (1987). La ecología del desarrollo humano. Barcelona: Paidós.

Dennison, P. (1997). Brain Gym. Aprendizaje de todo el cerebro. Robin Book.

Ecuador, M. d. (2014). Currículo de Educación Inicial. Quito.

Hall, G. y. (2011). Tratado de fisiología médica. Barcelona.

Hatfield. (25 de Marzo de 2006). Tony Hatfield's Retired Ramblings. Obtenido de http://retiredrambler.typepad.com/tonys_ramblings/2006/03/brain_gym_the_t.html

Ibarra, L. M. (2007). Aprende mejor con gimnasia cerebral. México: Mexicana.

Pérez-Cortés, E. M. (2003). Educación y estilos de aprendizaje-enseñanza. Mexico: Cuzco.

Perugachi, P. C. (2003). Gimnasia cerebral como técnica para producir cambios significativos en la lectura y escritura. Quito.

Rey, R. R. (2006). Funciones cerebrales superiores:. Revista de la Faculta de Medicina.

Rogoff, B. (1993). Aprendices del pensamiento: el desarrollo cognitivo en el contexto social. Paidós.

Schmid, A. \& Peper, E. (1991). Técnicas para el entrenamiento de la Concentración. En J.M. Williams: Psicología Aplicada al Deporte, Madrid

Schunk, D. H. (2012). Teorías del aprendizaje. Mexico.

Tinajero A. y Mustard, J. (2011). Reporte presentado a la BernardVan Leer Foundation, documento en proceso de publicacion.

Vergara Kliebs, M., \& Araya Castillo, M. (2005). "Gimnasia Cerebral en el aula".

Vygotski, L. (1979). El desarrollo de los procesos psicológicos superiores. México: Gijalbo. 
ANEXOS

\begin{tabular}{|c|c|c|c|}
\hline Título & Autores & $\begin{array}{l}\text { Metodología } \\
\text { aplicada }\end{array}$ & Resultados encontrados \\
\hline $\begin{array}{l}\text { "Gimnasia de pausa en el aula". } \\
\text { Ministerio de Educación, } \\
\text { Argentina, } 2005 .\end{array}$ & $\begin{array}{l}\text { Marlene Vergara } \\
\text { Kliebs y Mario } \\
\text { Araya Castillo, } \\
2005\end{array}$ & $\begin{array}{l}\text { Investigación cuasi- } \\
\text { experimental }\end{array}$ & $\begin{array}{l}\text { Entre los resultados se observan grandes } \\
\text { diferencias entre los grupos investigados } \\
\text { concluyendo que la investigación mejoró } \\
\text { considerablemente la agilidad mental, } \\
\text { vigilia y concentración del grupo } \\
\text { experimental ( Vergara Kliebs \& Araya } \\
\text { Castillo, 2005). }\end{array}$ \\
\hline $\begin{array}{l}\text { "La gimnasia cerebral como } \\
\text { técnica para producir cambios } \\
\text { significativos en la lectura y } \\
\text { escritura" } \\
\text { Universidad Politécnica } \\
\text { Salesiana, Ecuador, } 2003 \text {. }\end{array}$ & $\begin{array}{l}\text { Paulina Calderón } \\
\text { Perugachi }\end{array}$ & Ejercicios prácticos. & $\begin{array}{l}\text { Una vez realizado cada uno de ejercicios } \\
\text { la autora observa que más del } 50 \% \text { de los } \\
\text { estudiantes mejoraron la dificultad que } \\
\text { tenían con respecto a la lectoescritura, } \\
\text { por lo tanto, la autora concluye la } \\
\text { Gimnasia Cerebral ayuda } \\
\text { significativamente al proceso de } \\
\text { enseñanza aprendizaje, además, que el } \\
\text { movimiento es una de las técnicas más } \\
\text { sencillas, pero con valiosos resultados } \\
\text { para los estudiantes. (Perugachi, 2003). }\end{array}$ \\
\hline $\begin{array}{l}\text { Importancia de la Gimnasia } \\
\text { Cerebral en el desarrollo de la } \\
\text { creatividad de los niños y niñas } \\
\text { del primer año de educación } \\
\text { básica de la escuela Juan } \\
\text { Bautista Palacios "LA SALLE" } \\
\text { de la ciudad de Ambato en el } \\
\text { periodo lectivo 2010-2011 } \\
\text { Universidad Técnica de } \\
\text { Ambato, Ecuador, } 2012 .\end{array}$ & $\begin{array}{l}\text { Adriana Angélica } \\
\text { López Balcázar }\end{array}$ & $\begin{array}{l}\text { Investigación } \\
\text { exploratoria }\end{array}$ & $\begin{array}{l}\text { Los ejercicios de Gimnasia Cerebral en } \\
\text { el aula son una herramienta para } \\
\text { estimular y desarrollar habilidades y } \\
\text { capacidades cerebrales, además crea una } \\
\text { conexión entre cerebro y cuerpo a través } \\
\text { del movimiento, logrando armonía entre } \\
\text { aspectos emocionales, fisicos y mentales } \\
\text { permitiendo de esta manera dar rienda } \\
\text { suelta a la creatividad (Balcazar, 2012). }\end{array}$ \\
\hline
\end{tabular}

Tabla 1. Análisis y revisión de las investigaciones realizadas por los diferentes autores. 